\title{
An Automatic Localization of Optic Disc in Low Resolution Retinal Images by Modified Directional Matched Filter
}

\author{
Murugan Raman ${ }^{1}$, Reeba Korah ${ }^{2}$, and Kavitha Tamilselvan ${ }^{3}$ \\ ${ }^{1}$ College of Engineering Guindy, Anna University, India \\ ${ }^{2}$ Alliance College of Engineering and Design, Alliance University, India \\ ${ }^{3}$ New Prince Shri Bhavani College of Engineering and Technology, Anna University, India
}

\begin{abstract}
An automatic optic disc localization in retinal images used to screen eye related diseases like diabetic retinopathy. Many techniques are available to detect Optic Disc (OD) in high-resolution retinal images. Unfortunately, there are no efficient methods available to detect $O D$ in low-resolution retinal images. The objective of this research paper is to develop an automated method for localization of Optic Disc in low resolution retinal images. This paper proposes a modified directional matched filter parameters of the retinal blood vessels to localize the center of optic disc. The proposed method was implemented in MATLAB and evaluated both normal and abnormal low resolution retinal images using the subset of Optic Nerve Head Segmentation Dataset (ONHSD) and the success percentage was found to be an average of $96.96 \%$ with 23 seconds.
\end{abstract}

Keywords: Retinal image processing, dabetic retinopathy, optic disc, bood vessels, modified directional matched filter.

Received May 7, 2015; accepted October 7, 2015

\section{Introduction}

Automatic localization of retinal fundus images provides an efficient solution of screening for eye disease such as glaucoma, Diabetic Retinopathy (DR) $[1,3]$. Optic Disc (OD) localization is an integral part of the screening system for glaucoma $[8,11]$. OD detection is the first step to determine the retinal abnormalities, such as drusen, exudates, microaneurysms, and hemorrhage, and imaging artifacts, such as haze, lashes, and uneven illumination in the human eye $[10,16,20]$. The past OD detection methods are based on image characteristics, bright regions, regions with the highest image variation, anatomical structure and relative position between $\mathrm{OD}$ and macula. Jun Cheng et al. [4] employed super pixel classification and histogram based center surround statistics method for detection of OD. Principal Component Analysis (PCA) techniques are used by Mudassar and Butt [17] to segment OD and fovia. Suero et al. [22] proposed a method consisting of intensity of images that is resized and a shade-correction method for homogenizing the background and set of morphological opening and closing operations are used for optic disc localization in high resolution images.

Based on the above review most of the researchers considered the OD as the brightest region within retinal image. However, this criterion may not be applicable for dark diseased images like diabetic retinopathy.
Papers [2, 12, 21, 22] considered the OD as the area with highest variation in intensity of adjacent pixels. Both the criteria's are considered for normal, healthy high resolution retinal images. However, we found that all the OD detection algorithms often failed to prove dark, diseased low resolution retinal images. In our previous work [19] developed an approach to detect OD in digital color Images of the human retina using directional matched filter. This method is tested and is successful for high resolution retinal images and is failure for low resolution retinal images because of breaking of blood vessels (Figure 1-b). So we need to improve the performance of the matched filter and optimize better parameters for low resolution retinal images. Hence, this paper proposes to find better parameters and segments in non-breaking of Blood Vessels. (Figure1-c) for low resolution retinal images.

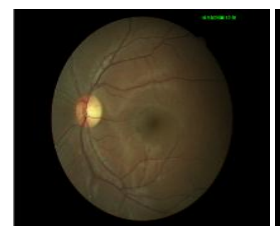

a) Low resolution retinal image source: ONHSD dataset.

b) Blood vessels low resolution images by our previous method (Breaking of blood vessels).

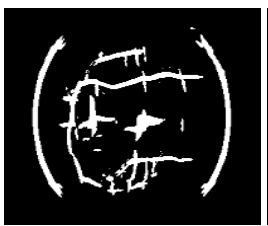

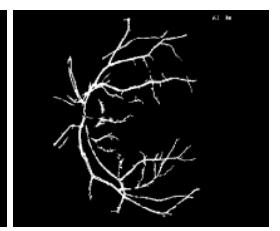

c) Blood vessels low resolution Images by proposed method (Non-breaking of Blood Vessels).
Figure 1. Performance of directional matched filter.

The aim of this paper is to develop an automatic detection of OD center in low resolution retinal 
images with high accuracy and less computational time. The remaining part of the paper organized as follows. In section 2 Materials and methodologies are presented. The results and discussion are presented in section 3 and Conclusion and future works are presented in section 4.

\section{Materials and Methodology}

\subsection{Low Resolution Retinal Fundus Image Dataset}

The low resolution retinal digital fundus images were collected form Optic Nerve Head Segmentation Dataset (ONHSD) [13]. This dataset contains 99 fundus images taken from 50 patients randomly sampled from a diabetic retinopathy screening programme. The images are various ethnic backgrounds (Asian 20\%, A fro-Caribbean 16\%, Caucasian 50\%, Unknown 14\%). The images were acquired using a Canon CR6 45MNf fundus camera, with a field angle lens of 45 degrees, resolution $640 \times 480$ (about 20 per pixel). We identified this as the only data set for low resolution since Low resolution image intensity range is rather narrow, it does not cover the potential range of [0-255] and the high resolution image covers the range [0-255].

\subsubsection{Green Band Equalization (GBE)}

The retinal images are non-uniform due to uneven illumination. To eliminate the non-uniform illumination Hoover and Goldbaum [9] equalized each pixel using the following Equation (1)

$$
\mathrm{I}_{g}(\mathrm{r}, \mathrm{c})=\mathrm{I}(\mathrm{r}, \mathrm{c})+\mathrm{m}-\mathrm{I}_{w}(\mathrm{r}, \mathrm{c})
$$

Where $I_{g}(r, c)$ is intensity of the pixels, $\mathrm{m}$ is desired average Intensity (128-8 bit gray scale image). $I_{w}(r$, c) mean intensity value of the pixels within a window ' $\mathrm{w}$ ' of size $\mathrm{N} x \mathrm{~N}$. We used running window of size 40x40 as applied by Walter and Klein [23]. The GBE image is in Figure 2-b.

\subsubsection{Adaptive Histogram Equalization (AHE)}

AHE is a computer image processing technique used to normalize and enhance the contrast within the fundus image. It differs from histogram equalization when detecting low resolution levels. AHE is applied to an GBE as proposed in $\mathrm{Wu}$ et al. [25], where each pixel ' $p$ ' is adapted using the following Equation:

$$
\mathrm{I}_{\mathrm{AHE}}(p)=\left(\sum_{p^{\prime} \in R(p)} \frac{s\left(I(p)-I\left(p^{\prime}\right)\right.}{h^{2}}\right)^{r} * \mathrm{M}
$$

Where $M=255, R(p)$ is the pixel p's neighborhood and $h$ is the square window length which is 81 . The AHE image is shown in Figure 2-c.

\subsubsection{Contrast Limited Adaptive Histogram Equalization (CLAHE)}

Contrast Limited Adaptive Histogram Equalization (CLAHE) enhances the resolution of the grayscale image by transforming the values. It operates on small regions in the image, called tiles, rather than the entire image. Each tile's resolution is enhanced, so that the histogram of the output region approximately matches the histogram specified by the distribution parameter. The neighbouring tiles are then combined using bilinear interpolation to eliminate artificially induced boundaries. The contrast, especially in homogeneous areas, can be limited to avoid amplifying any noise that might be present in the image. The contrast amplification in the vicinity of a given pixel value is given by the slope of the transformation function. This is proportional to the slope of the labelled OD Cumulative Distribution Function (CDF) and therefore to the value of the histogram at that pixel value [18]. The CLAHE image is in Figure 2-d.

\subsubsection{Blood Vessels Segmentation by Modified Directional Matched Filter}

The matched filter is one of the template matching method which is used to detect Blood Vessel in retinal fundus images. The matched filter uses number of samples taken across a section of retinal Blood Vessels [9]. A prototype matched filter is expressed as

$$
\mathrm{k}(\mathrm{x}, \mathrm{y})=-\exp \left(-\mathrm{x}^{2} / 2 \sigma^{2}\right) \forall|\mathrm{y}| \leq \mathrm{L} / 2,
$$

Where $L$ is the length of the vessel segment that has the same orientation, $\sigma$ defines the spread of the intensity profile. To be able to detect vessels on all possible orientations, the kernel must be rotated to all possible vessel orientations and the maximum response from the filter bank is registered. Many papers found that rotating by an amount of $15^{\circ}$ is adequate to detect vessels with an acceptable amount of accuracy which results in a filter bank with 12 kernels. The values of $L$ and $\sigma$ found that the best parameter values were those that gave the maximum response at $L=9$ and $\sigma=2$. However, we present our experiments of finding $L$ and $\sigma$. A Gaussian curve has infinitely long double sided trails; the trails are truncated at $u= \pm 3 \sigma$. A neighborhood $N$ is defined such that

$$
\mathrm{N}=\{(\mathrm{u}, \mathrm{v}),|\mathrm{u}| \leq \mathrm{T},|\mathrm{v}| \leq \mathrm{L} / 2\}
$$

Where $T=3 \sigma$. Let Pi be the points that belongs to the neighbourhood $\mathrm{n}$ given as

$$
\mathrm{p}_{i}[\mathrm{u}, \mathrm{v}]=[\mathrm{x}, \mathrm{y}]\left[\begin{array}{cc}
\cos \theta & -\sin \theta \\
\sin \theta & \cos \theta
\end{array}\right]
$$


The corresponding weights in the kernel $i(i=1, \ldots, 12$ which is the number of kernel) are given by

$$
\mathrm{k}_{i}(\mathrm{x}, \mathrm{y})=-\exp \left(-\mathrm{u}^{2} / 2 \sigma^{2}\right) \forall \mathrm{pi} \in \mathrm{N}
$$

The filter is normalized to have zero mean as follows

$$
\mathrm{k}_{i}^{\prime}(\mathrm{x}, \mathrm{y})=\mathrm{k}_{i}(\mathrm{x}, \mathrm{y})-\mathrm{m}_{i},
$$

Where $\mathrm{m}_{i}=1 / \mathrm{a} \sum_{p_{i} \mathrm{EN}} \mathrm{k}_{i}(\mathrm{x}, \mathrm{y})$ and ' $\mathrm{a}$ ' denotes the number of points in $\mathrm{N}$.

\subsubsection{Modification Parameters}

To improve the performance of the matched filter we need to find better parameters for $L, \sigma$, and $T$ that could be suitable for low resolution images. The optimization program is simple in which we use an exhaustive search for the best parameters of $\mathrm{L}, \sigma$ and $\mathrm{T}$. The search space is not very large since we limit the values of those parameters to $\mathrm{L}=\{7,7.1,7.2, \ldots, 11\}, \quad \sigma=\{1.5,1.6,1.7, \ldots, 3\}, \mathrm{T}=\{2$, $2.25,2.5, \ldots, 10\}$. Let the input retina image be $f$ and the output filtered image be ' $\mathrm{fL} \sigma \mathrm{T}$ ' to decide whether a filtered image is good or bad, it is threshold according to Otsu [18] yielding ' $\mathrm{gL} \sigma \mathrm{T}$ ' and then compared to a corresponding hand labelled retina $\mathrm{h}$ image. The hand labelled image is obtained from a retina image by an experienced observer to be used for comparison purposes [23].The final segmented blood vessel is shown in Figure 2-e). For low resolution retinal image containing vessels of varying width, we find better parameters for $\mathrm{L}, \sigma$, and $\mathrm{T}$ for a smaller value for small vessels and a larger value for the large vessels. The blood vessel removed images are shown in Figure 2-f.

\subsubsection{Optic Disc Localization}

Next, we performed a column wise neighbourhood operation. A 9x9 template (Table 1) matrix is created that has a separate column for each pixel in the original image. The column corresponding to a given pixel contains the values of that pixel's neighbourhood from the original image.

Table 1. Template matrix.

\begin{tabular}{|c|c|c|c|c|c|c|c|c|}
\hline 135 & 120 & 105 & 105 & 90 & 75 & 75 & 60 & 45 \\
\hline 150 & 135 & 120 & 105 & 90 & 75 & 60 & 45 & 30 \\
\hline 165 & 150 & 135 & 120 & 90 & 60 & 45 & 30 & 15 \\
\hline 165 & 165 & 150 & 135 & 90 & 45 & 30 & 15 & 15 \\
\hline 0 & 0 & 0 & 0 & 90 & 0 & 0 & 0 & 0 \\
\hline 15 & 15 & 45 & 60 & 90 & 120 & 135 & 150 & 165 \\
\hline 15 & 30 & 45 & 60 & 90 & 120 & 135 & 150 & 165 \\
\hline 30 & 45 & 60 & 75 & 90 & 105 & 120 & 135 & 150 \\
\hline 45 & 60 & 75 & 75 & 90 & 105 & 105 & 120 & 135 \\
\hline
\end{tabular}

To reduce the computational burden, matched filters are applied only to candidate pixels picked from the fundus image. The binary vessel image is thinned. Hence, reducing the amount of pixels labelled as vessels into the vessel's centerline. Next, a column wise neighbourhood operation removed the background, leaving only the border edge effect from thinned image. Next, we performed thresholding on the image. We used disc-shaped structuring element of size 5 and performed morphological closing. The optic disc is the part of the retina which has the highest pixel value. Hence, to locate the optic disc, we had to detect the edges first and then created the region of interest (mask) as shown in the Figure 2-g). We have chosen the radius of optic disc to be 30. Further, the optic disc mask is superimposed with the vessel removed image (Figure 2-f) to localize the optic disc (Figure 2-h).

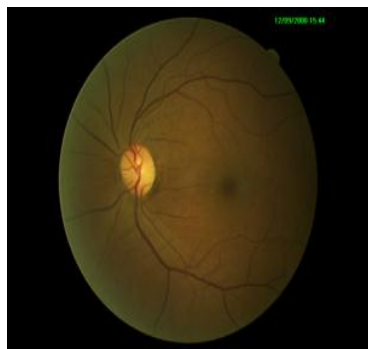

a) Input image.

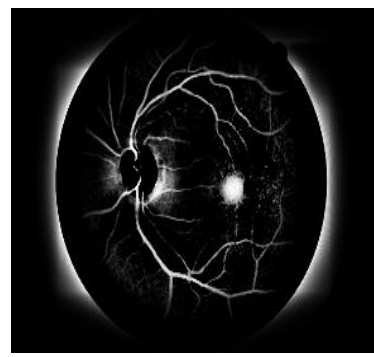

c) Adaptive Histogram equalized image.

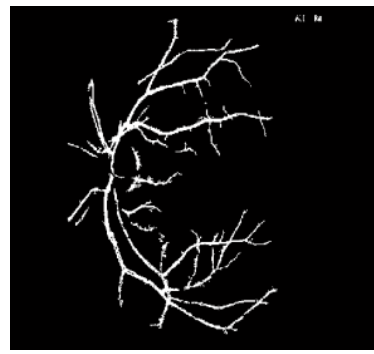

e) Blood vessel segmented image.

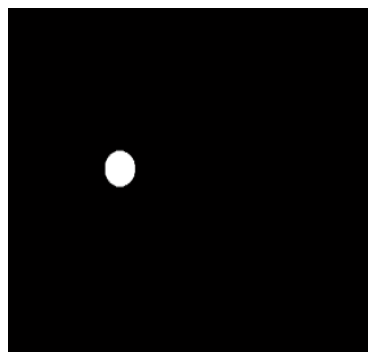

g) Masking of OD.

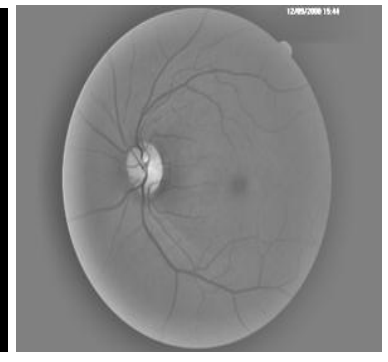

b) Green band equalized image.

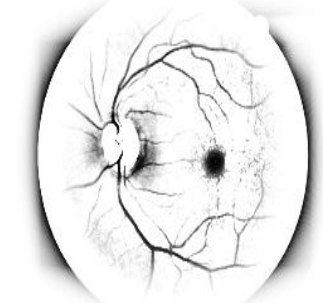

d) Contrast limited adaptive histogram equalized image.

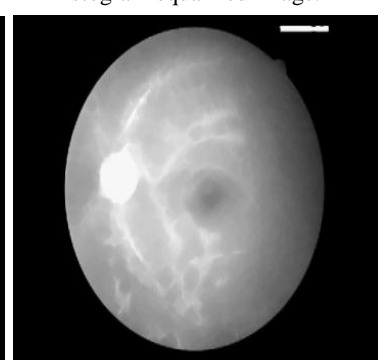

f) Blood vessels removed image.

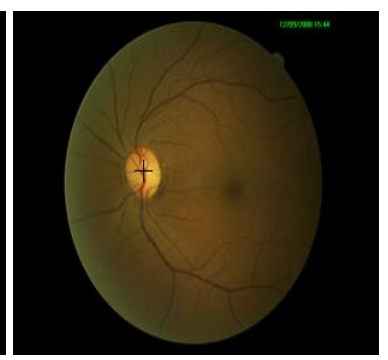

h) OD detected image (Block cross.
Figure 2. Optic Disc localization results.

\section{Results and Discussion}

The proposed method was implemented in MATLAB R2013 where runs needed an average of 23 seconds for each image on a laptop Pentium (R) Dual-Core2 CPU 
and T4300@ 2.10GHz, 3.00 GB RAM 64-bit OS). The proposed method was evaluated both normal and abnormal low resolution retinal images using the subset of the ONHDS and the success percentage was found to be $96.96 \%$. The optic disc center is located correctly in 96 out of 99 images (Normal: 30, Glaucoma: 38, DR: 31). Figure 3 shows results for random samples of success images.
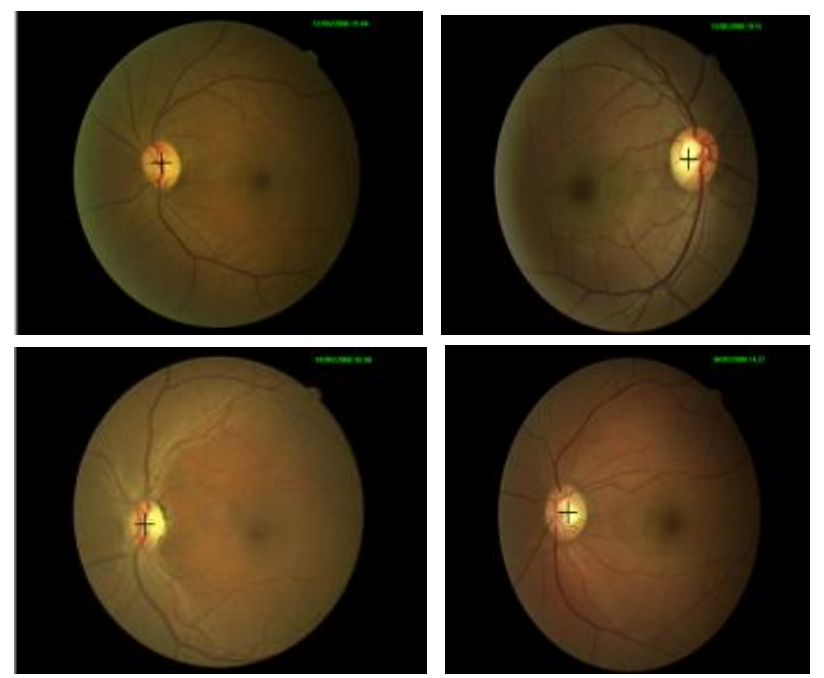

Figure 3. Results of the proposed method (Black Cross represent the estimated OD Center) form ONH Dataset.

Accuracy of proposed approach, 96.96\%, is comparable to other state-of-the-art approaches (shown in Figure 4). Though, Lowell et al. [13] was able to achieve slightly higher accuracy than the proposed approach, it has to be noted that his approach depends on various parameters which need to be tuned to achieve high accuracies. Whereas the proposed approach does not have any parameters to be tuned. Dashtbozorg et al. [5] achieved slightly lower accuracy, due to the lack of structural information at the OD location.

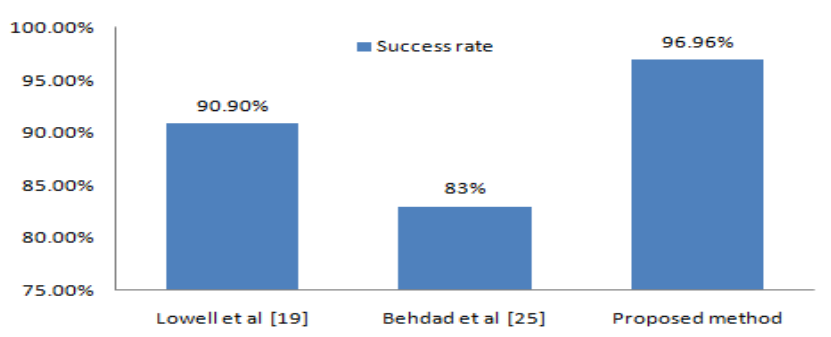

Figure 4. Accuracy of proposed OD detection method with other state-of-the-art approaches.

The computation time (in seconds) of proposed approach is least when compared to other state of the art methods, as shown in Table 2. After finalizing the $\mathrm{OD}$ detection the results were compared with those of the Circular Hough Transform [2], Morphology-based method [22], Temporal Lock [12], DV-Hough methods [15]. The mean and the standard deviations values were calculated for ONHD. For comparing our results with those of the other methods, we have used the same subjective evaluation method defined by Lowell et al. [13]. The discrepancy, $\delta_{\mathrm{j}}$ on image $\mathrm{j}$ is defined by.

Table 2. Comparison of complexity.

\begin{tabular}{|c|c|c|}
\hline Authors & Success rate $\mathbf{( \% )}$ & $\begin{array}{c}\text { Computation } \\
\text { time (Sec) }\end{array}$ \\
\hline Dashtbozorg $\boldsymbol{e t}$ al. $\cdot[\mathbf{5}]$ & 86 & 10.6 \\
\hline Mary $\boldsymbol{e t}$ al.[15] & 94 & 1.1 \\
\hline Hoover and Goldbaum [9] & 89 & 1.5 \\
\hline Lalonde $\boldsymbol{e t}$ al.[12] & 71.6 & 1.6 \\
\hline Wisaeng $\boldsymbol{e t}$ al.[24] & 91.35 & 34 \\
\hline Dehghani $\boldsymbol{e t}$ al.[6] & 93 & 40 \\
\hline Dehghani et al.[7] & 96 & 27.6 \\
\hline Mahfouz and Fahmy [14] & 92.6 & 46 \\
\hline Proposed method[a] & 96.96 & 23 \\
\hline
\end{tabular}

[a]-our proposed method result is shown in the last row with highest success percentage with less computation time.

$$
\delta_{j}=\sum_{i} \frac{\left|m_{i}^{j}-\mu_{i}^{j}\right|}{\sigma_{i}^{j}+\varepsilon}
$$

Where $\mu_{\mathrm{i}}^{\mathrm{j}}$ and $\sigma_{\mathrm{i}}^{\mathrm{j}}$ are, respectively, the mean and the standard deviation of values obtained for $i$ of image $\mathrm{j}, \mathrm{m}_{\mathrm{i}}^{\mathrm{j}}$ is the location of the OD $\mathrm{i}$ of image $\mathrm{j}$ and $\epsilon$ is a small factor to prevent division by zero and agreement is set equal to 0.5 .

Lowell et al. [13] also classified each image based on the discrepancy value as Excellent $\left(\delta_{\mathrm{j}}<1\right)$, Good $\left(\delta_{\mathrm{j}}<2\right)$, Fair $\left(\delta_{\mathrm{j}}<5\right)$, and Poor $\left(\delta_{\mathrm{j}} \geq 5\right)$. The localization methods are assessed considering the percentage of OD localizations classified into those four categories.

Table 3 shows the success percentage of images per subjective category for different methods. The results of the proposed method are included with other state of the art methods.

Table 3. Comparison between proposed method and four other methods in terms of percentage images per subjective category (ONHSD).

\begin{tabular}{|c|c|c|c|c|c|}
\hline Method & Excellent & Good & Fair & Poor & $\begin{array}{c}\text { Excellent- } \\
\text { Fair }\end{array}$ \\
\hline $\begin{array}{c}\text { Circular Hough } \\
\text { transform[2] }\end{array}$ & 40 & 39 & 18 & 3 & 97 \\
\hline Morphology[22] & 28 & 36 & 31 & 6 & 94 \\
\hline $\begin{array}{c}\text { Temporal } \\
\text { Lock[12] }\end{array}$ & 42 & 31 & 10 & 17 & 83 \\
\hline DV-Hough[15] & 39 & 22 & 20 & 19 & 81 \\
\hline Proposed & 44 & 47 & 9 & 0 & 100 \\
\hline
\end{tabular}

In order to be able to compare with other published works, other measures were calculated: Accuracy (ACC) which is calculated by the sum of correctly detected OD pixels and non-OD pixels divided by the total number of pixels; True Positive Fraction (TPF) is obtained by dividing the number of correctly detected OD pixels by the total number of OD pixels in the reference image; False Positive Fraction (FPF) which is the ratio between the number of wrong detected OD pixels and the total number of non-OD pixels in the reference image; 
the mean absolute distance (MAD) which measures the accuracy of the OD location. Figure 5 compares the result of our method with the results of morphology on the ONHSD.
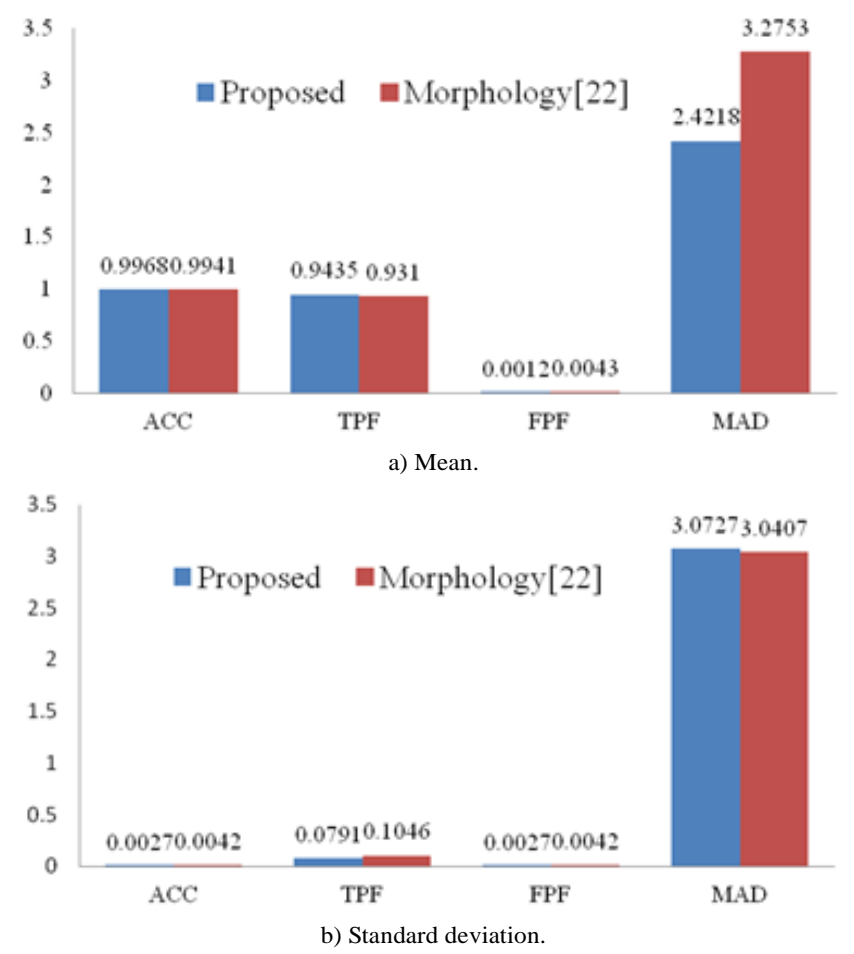

Figure 5. Comparison of different measures between proposed method and morphological methods on ONHSD dataset.

\section{Conclusions}

This paper has presented a most efficient technique for OD localization from low resolution retinal fundus images using modified directional matched filter. The proposed technique produced better results from publicly available low resolution Optic Nerve Head Dataset (ONHDS). The proposed method has following advantages,

- Tolerant to the low resolution retinal images.

- The designed filter is stable and easy for implementation.

- The designed filter can be extended for macula detection with little adaptation.

Thus, the automated method is useful for the following real time applications.

- Localizing and segmenting retinal structure such as blood vessels, macula and fovia and also other anatomical structure such as microaneurysms, hemorrhage, drusen and exudates.

- To classify left and right eyes in macula-centered retinal images

- OD localization is a first key processing component in many algorithms designed for the automatic extraction of retinal abnormalities

- The OD was also used as an initial point for retinal vasculature tracking methods.
Thus the proposed approach helps to ophthalmologists in screening process of retinal image analysis and other eye related screening processes.

\section{References}

[1] Aquino A., Gegundez-Arias M., and Marín D., "Detecting the Optic Disc Boundary In Digital Fundus Images Using Morphological, Edge Detection, and Feature Extraction Techniques," IEEE Transactions on Medical Imaging, vol. 29, no. 11, pp. 1860-1869, 2010.

[2] Belhadi S. and Benblidia N., "Automated Retinal Vessel Segmentation using Entropic Thresholding Based Spatial Correlation Histogram of Gray Level Images," The International Arab Journal of Information Technology, vol. 12, no. 5, pp. 441-447, 2015.

[3] Chaudhuri S., Chatterjee S., Katz N., Nelson M., and Goldbaum M., "Detection of Blood Vessels in Retinal Images Using Two Dimensional Matched Filters," IEEE Transactions Medical Imaging, vol. 8, no. 3, pp. 263-269, 1989.

[4] Cheng J., Liu J., Xu Y., Yin F., Wong D., Tan N., Tao D., Cheng C., Aung T., and Wong T., "Superpixel Classification based Optic Disc and Optic Cup Segmentation for Glaucoma Screening," IEEE Transactions on Medical Imaging, vol. 32, no. 6, pp. 1019-1032, 2013.

[5] Dashtbozorg B., Mendonça A., and Campilho A., "Optic Disc Segmentation Using The Sliding Band Filter," Computers in Biology and Medicine, vol. 56, pp. 1-12, 2015.

[6] Dehghani A., Moghaddam H., and Moin M., "Optic Disc Localization in Retinal Images Using Histogram Matching," EURASIP Journal on Image and Video Processing, vol. 1, no. 19, pp. 1-11, 2012.

[7] Dehghani A., Moin M., and Saghafi M., "Localization of the Optic Disc Center in Retinal Images Based on the Harris Corner Detector," Biomedical Engineering Letters, vol. 2, no. 3, pp. 198-206, 2012.

[8] Haseeb A., "Diabetic Retinopathy-Biomolecules and Multiple Pathophysiology," Diabetes and Metabolic Syndrome: Clinical Research and Reviews, vol. 9, no. 1, pp. 51-54, 2015.

[9] Hoover A. and Goldbaum M., "Locating the Optic Nerve in A Retinal Image Using the Fuzzy Convergence of the Blood Vessels," IEEE Transactions on Medical Imaging, vol. 22, no. 8, pp. 951-958, 2003.

[10] Khachatryan N., Bowd C., Medeiros F., and Zangwill L., "Optic Disc Imaging," Glaucoma, vol. 1, pp. 221-243, 2015.

[11] Krishnan M., Acharya R., Chua C., Min L., Ng E., Mushrif M., and Laude A., "Application of 
Intuitionistic Fuzzy Histon Segmentation for the Automated Detection of Optic Disc in Digital Fundus Images," in Proceedings of IEEE-EMBS International Conference on Biomedical and Health Informatics, Hong Kong, pp. 444-447, 2012.

[12] Lalonde M., Beaulieu M., and Gagnon L., "Fast and Robust Optic Disk Detection Using Pyramidal Decomposition and Hausdorff-Based Template Matching," IEEE Transactions on Medical Imaging, vol. 20, no. 11, pp. 1193-1200, 2001.

[13] Lowell A., Hunter D., Steel A., Basu R., Ryder E., Fletcher., and Kennedy L., "Optic Nerve Head Segmentation," IEEE Transactions on Medical Imaging, vol. 23, no. 2, pp. 256-264, 2004.

[14] Mahfouz F. and Fahmy A., "Fast Localization of the Optic Disc Using Projection of Image Features," IEEE Transactions on Image Processing, vol. 19, no. 12, pp. 3285-3289, 2010.

[15] Mary C., Rajsingh E., Jacob K., Anandhi D., Amato U., Selvan S., Anandhic D., Amato U., and Selvan E., "An Empirical Study on Optic Disc Segmentation Using an Active Contour Model," Biomedical Signal Processing and Control, vol. 18, pp. 19-29, 2015.

[16] Morales S., Naranjo V., Angulo J., and Alcaniz M., "Automatic Detection of Optic Disc Based on PCA and Mathematical Morphology," IEEE Transactions on Medical Imaging. vol. 32, no. 4, pp. 786-796, 2013.

[17] Mudassar A. and Butt S., "Application of Principal Component Analysis in Automatic Localization of Optic Disc and Fovea in Retinal Images," Journal of Medical Engineering, vol. 2013, pp. 1-12, 2013.

[18] Otsu N., "A Threshold Selection Method from Gray Level Histograms," IEEE Transactions on Systems, Man, and Cybernetics, vol. 9, no. 1, pp. 62-66, 1979.

[19] Raman M. and Korah R., "An Efficient Approach to Detect Optic Disc in Digital Color Images of the Human Retina," CIIT International Journal of Digital Image Processing, vol. 3, no. 12, pp. 771-774, 2011.

[20] Scanlon P., "Diabetic Retinopathy," Medicine, vol. 43, no. 1, pp. 13-19, 2015.

[21] Staal J., Abramoff M., Niemeijer M., Viegever M., and Ginneken B., "Ridge Based Vessel Segmentation in Color Images of the Retina," IEEE Transactions on Medical Imaging, vol. 23 no. 4, pp. 501-509, 2004.

[22] Suero A., Marin D., Arias M., and Bravo J., "Locating the Optic Disc in Retinal Images Using Morphological Techniques," in Proceedings of IWBBIO, Granada, pp. 593-600, 2013.
[23] Walter T. and Klein J., "Segmentation of Color Fundus Images of The Human Retina: Detection Of The Optic Disc and the Vascular Tree Using Morphological Techniques," in Proceedings of International Symposium on Biological and Medical Data Analysis, Madrid, pp. 282-287, 2001.

[24] Wisaeng K., Hiransakolwong N., and Pothiruk E., "Automatic Optic Disc Detection from Low Contrast Retinal Images," Applied Mathematical Sciences, vol. 6, no. 103, pp. 5127-5136, 2012.

[25] Wu D., Zhang M., Liu J., and Bauman W., "On the Adaptive Detection of Blood Vessels in Retinal Images," IEEE Transactions on Biomedical Engineering, vol. 53, no. 2, pp. 341343, 2006. 


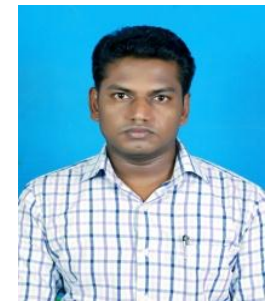

Murugan Raman has completed his M.E. degree in Embedded System Technologies from Anna University, Coimbatore, India and his B.E.Degree in Anna University Chennai, India. Currently he is pursuing his $\mathrm{Ph} . \mathrm{D}$ in Anna University, Chennai for the field of Retinal Image analysis. He has 10 years experience in teaching. He is working as Assistant Professor in AMS College of Engineering, Avadi-IAF, Chennai. He published 6 International Journals and presented 5 national conferences \& 6 international conference. His research interest includes medical Imaging and Signal Processing.

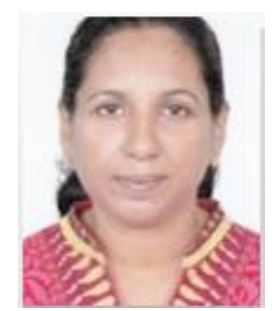

Reeba Korah teaches subjects in Electronics and Communication Engineering and pursues scholarly research in low power VLSI and wireless sensor networks. An alumna of Marathwada University, Aurangabad, Maharastra and Anna

University, Chennai, Professor Korah holds a doctoral degree in VLSI and Video Processing from Anna University, Chennai. An accomplished scholar, Professor Korah has published extensively in international, peer reviewed journals, and supervised doctoral scholars.

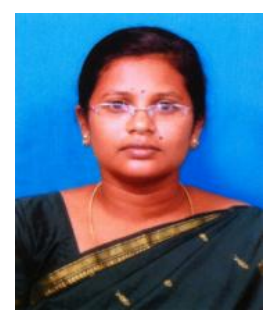

Kavitha Tamilselvan completed her Ph.D in Anna University Chennai, India. She received her M.E. degree in Systems Engineering and Operations Research from Anna University, Chennai. B.E. in Electronics and Communication Engineering from Bharathidasan University, India. Presently she is working as professor in New Prince Shri Bhavani College of Engineering and Technology. Her fields of interests are Wireless Networks, Wireless Sensor Network and information security, etc. She has published 20 papers in national/International conferences and 10 in International Journals. 